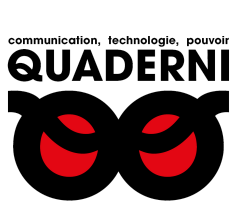

\title{
Quaderni
}

Communication, technologies, pouvoir

94 | Automne 2017

Les théories du complot à l'heure du numérique

\section{Vanessa Ogle, The Global Transformation of Time:}

$1870-1950$

\section{Côme Souchier}

\section{(2) OpenEdition}

10 Journals

\section{Édition électronique}

URL : https://journals.openedition.org/quaderni/1120

DOI : 10.4000/quaderni. 1120

ISSN : 2105-2956

Éditeur

Les éditions de la Maison des sciences de l'Homme

\section{Édition imprimée}

Date de publication : 5 octobre 2017

Pagination : 101-104

\section{Référence électronique}

Côme Souchier, «Vanessa Ogle, The Global Transformation Of Time: 1870-1950 », Quaderni [En ligne], 94 | Automne 2017, mis en ligne le 05 octobre 2017, consulté le 23 juillet 2021. URL : http:// journals.openedition.org/quaderni/1120; DOI : https://doi.org/10.4000/quaderni. 1120 


\section{Compte rendu}

\section{The Global Transformation \\ Of Time: 1870-1950 \\ Vanessa Ogle}

Harvard University Press : Cambridge, Massuchusetts, London, England, 2015

par Côme Souchier

Sciences Po Grenoble

La seconde moitié du XIX ${ }^{\mathrm{e}}$ siècle est marquée par les débuts d'une interconnexion mondiale à une échelle inédite grâce aux réseaux télégraphiques, aux chemins de fer et aux bateaux à vapeur. De nombreux intellectuels, hommes politiques, journalistes et scientifiques participent alors d'une vaste réflexion sur la mesure et l'emploi du temps à une époque où la comparaison entre sociétés vire parfois à l'obsession (p. 5). Vanessa Ogle, historienne et professeur assistante à l'Université de Pennsylvanie, multiplie les terrains d'étude pour saisir la «transformation globale du temps » nourrie par cette activité intellectuelle en Europe, en Inde, au Proche-Orient et en Amérique du nord. Son livre atteste de la pertinence d'une approche globale de l'histoire contemporaine, tant l'analyse en termes de circulation des personnes, des biens et des idées s'avère féconde pour appréhender des phénomènes irréductibles aux seuls prismes du local ou du national. Mais l'intérêt de l'ouvrage repose surtout dans son appréciation fine des ressorts de la globalisation, ne réduisant pas cette dernière à un simple mouvement d'homogénéisation.

\section{La conscience du global}

L'effort comparatiste fourni par l'auteure lui permet en effet de relativiser l'interprétation diffusionniste qui consiste à faire du « temps global » une invention européenne adoptée par le reste du monde au profit d'une analyse en termes d'émulations et de similarité des conditions d'émergence de nouveaux rapports au temps. Le livre se propose d'étudier « comment les perceptions et les appréhensions d'un monde interconnecté ont fait émerger la politique de réforme du temps global, et comment le temps a servi d'outil 
intellectuel et institutionnel pour imaginer le monde comme étant global et interconnecté » (p. 206). Vanessa Ogle met ainsi en évidence la dualité du rôle social des repères temporels comme l'heure ou le calendrier, à la fois facteur d'unification (de la nation, du monde globalisé, de l'Oumma) et vecteur de différenciation (entre nations, cultures ou civilisations).

L'auteur retrace, par exemple, l'adoption des heures nationales en Angleterre (1848), aux États-Unis (1883), en France (1891) et en Allemagne (1893) : si chaque pays en vient à se saisir de la question horaire en réponse au développement des transports et des communications à l'échelle de son territoire, la connaissance de ce qui se fait ailleurs nourrit le sentiment d'une urgence à réformer le système horaire pour répondre aux besoins d'un monde connecté et compétitif dans lequel le temps, et la rationalisation de sa gestion, semblent gager de la puissance nationale (« Chapter One. National Times in a Globalizing World »).

Si les limites linguistiques et logistiques de l'auteur expliquent probablement le choix des terrains retenus et l'exclusion, notamment, de l'Amérique latine et d'une partie de l'Asie, on regrette que ces choix ne fassent pas l'objet d'un moment réflexif. Pour se rapprocher de l'exhaustivité spatiale à laquelle invite l'histoire globale, et pour bien cerner les contours d'une conscience globale d'un objet, ici le temps, il faudrait être capable de « suivre » cet objet dans tous les espaces linguistiques qu'il traverse et dont il émerge. Une telle histoire nécessite, à défaut d'un réel projet collectif et international, du moins des travaux qui se répondent et se coordonnent. On touche probablement, dans cet ouvrage, aux limites de ce que peut faire un chercheur dans une démarche solitaire d'histoire globale.

\section{Une standardisation du temps inaboutie}

Après l'unification des heures nationales, l'adoption progressive à partir des années 1880 d'un système de fuseaux horaires fondé sur le méridien de Greenwich marque un nouveau stade de la standardisation du temps à l'échelle mondiale (« Chapter Three. From National to Uniform Time around the Globe »). Ce n'est toutefois qu'au lendemain de la Seconde Guerre mondiale que le système des fuseaux horaires se stabilise (p. 96). Vanessa Ogle explore également comment le monde musulman a intégré les nouvelles techniques de communication et le rapprochement spatio-temporel qu'elles permettent 
1. Voir D. S. Landes, L'heure qu'il est. Les horloges, la mesure $d u$ temps et la formation du monde moderne, Paris, Gallimard, 1987, 622 p. ; G. Dohrn-Van Rossum, L'histoire de l'heure : l'horlogerie et l'organisation moderne du temps, Première édition., Paris, Éditions de la Maison des sciences de l'homme, 1997, 466 p. ; Howse Derek, Greenwich Time and the Longitude, Oxford University Press, Oxford, Philip Wilson Publishers Limited, 1997.

2. E. P. Thompson, « Time, Work-Discipline, and Industrial Capitalism ", Past \& Present, 1967, n० 38 , pp. 56-97. entre des sociétés jusque-là dispersées, pour tenter de définir un calendrier commun au début du XX ${ }^{\mathrm{e}}$ siècle (« Chapter Six. Islamic Calendar Times »). Vanessa Ogle retrace enfin l'histoire peu connue des tentatives d'instauration d'un calendrier universel, notamment via la Société des Nations, comme autant de traces d'une « ideology of universal time unification » portée par des milieux réformateurs hétéroclites où se côtoient scientifiques et industriels («Chapter Seven. One Calendar for All »). Ces projets de réformes, fondés le plus souvent sur le calendrier grégorien, suscitent scepticisme et opposition de la part d'autorités religieuses soucieuses de maintenir la dimension sacrée des rythmes calendaires (pp. 196-199). L'homogénéisation de la mesure du temps ne s'accomplit d'ailleurs jamais entièrement, en témoigne aujourd'hui encore la coexistence des calendriers musulmans, grégoriens et chinois, les arrangements avec les fuseaux horaires, ou encore les débats sur l'heure d'été.

L'ouvrage de Vanessa Ogle aborde principalement le temps par le biais de ses instruments de mesure (l'heure et le calendrier), ce qui le rapproche d'une histoire bien établie de la mesure du temps ${ }^{1}$. Mais il cherche également à dépasser cette histoire en intégrant ces instruments dans une réflexion plus large sur le rapport au temps des sociétés. Le manque de conceptualisation des phénomènes étudiés ensemble sous le terme de "transformation globale du temps » limite toutefois la portée de cet effort. Si la mise en commun du «temps social» (p. 47), de la « mesure du temps», ou encore de la « discipline temporelle » (pp. 71-74) paraît fondée, notamment parce que les sources de l'époque témoignent de cette intrication, elle devrait être plus amplement justifiée et expliquée.

\section{Le bon emploi de son temps}

La notion de temps social, " the useful deployment and division of society's times-work time, leisure, and recreation » (p. 47) est abordée par l'auteur à propos de la question de l'heure d'été (« Chapter Two. Saving Social Time »). Vanessa Ogle montre de façon convaincante que, contrairement à l'idée couramment admise à la suite des travaux d'E. P. Thompson selon lesquels l'abstraction du temps était déjà aboutie au début du $\mathrm{XIX}^{\mathrm{e}}$ siècle $^{2}$, la représentation " naturaliste » et « essentialiste » du temps a perduré en Europe jusqu'au milieu du $\mathrm{XX}^{\mathrm{e}}$ siècle. La résistance à l'abstraction du temps nourrit en tout cas les incompréhensions vis-à-vis des réformes de l'heure d'été à partir de la Première Guerre mondiale, accusées de remettre 
en cause le cycle naturel des hommes rythmé par le mouvement du soleil. Les avocats de l'heure d'été justifient en retour la réforme au nom d'une économie du temps et de sa gestion rationnelle à des fins progressistes.

C'est justement cette gestion rationalisée du temps que des intellectuels de Beyrouth cherchent à promouvoir pour rattraper ce qu'ils estiment être leur « retard » par rapport aux puissances occidentales (« Chapter Five. Comparing Time Management »). À l'origine de nombreuses réflexions sur le temps et sa nature, ce contact entre différentes cultures et civilisations amène soit le dialogue, l'imitation, l'adaptation, comme dans le cas de Beyrouth, ou alors l'aliénation, le ressentiment et la confrontation, comme dans le cas indien : les tentatives britanniques d'imposer le système horaire de Greenwich à Bombay à la fin du XIX ${ }^{\mathrm{e}}$ siècle se sont en effet heurtées à de vives résistances de la part de la population indienne, dont la mobilisation sur la question horaire s'inscrit dans le développement d'un sentiment anticolonial et national (« Chapter Four. A Battle of Colonial Times »).

Si l'ouvrage ne se revendique pas d'une approche socio-historique du politique mais d'une approche globale de l'Histoire, il peut contribuer à la compréhension de phénomènes et d'objets généralement associés à la première, comme la construction et le fonctionnement de l'État, la configuration entre science, savoir et gouvernement, ou encore la sociogenèse des nationalismes. Son livre participe également d'un questionnement fécond qui parcourt les travaux sur la globalisation contemporaine, à savoir l'articulation entre intégration mondiale d'une part, et montée des nationalismes d'autre part ${ }^{3}$. Il représente surtout un travail précieux pour la recherche sur l'organisation sociale du temps et l'organisation temporelle du social, aujourd'hui dispersées entre sociologie des temps sociaux, histoire des sensibilités, épistémologie de l'histoire et philosophie.

\footnotetext{
3. Cette question est notamment soulevée par S. Kern, The Culture of Time and Space 18801918, Cambridge, Massachusetts, Harvard University Press, 1983, $372 p$.
} 